\title{
Co-culture of peripheral blood mononuclear cell (PBMC) and human coronary artery endothelial cell (HCAEC) reveals the important role of autophagy implicated in Kawasaki disease
}

\author{
Jie Qin ${ }^{1,2 \#}$, Yiming Zheng ${ }^{1 \#}$, Yueyue Ding ${ }^{1}$, Chengcheng Huang ${ }^{3}$, Miao Hou ${ }^{1}$, Mei Li ${ }^{4}$, Guanghui Qian ${ }^{1,4}$, \\ Haitao $\mathbf{L v}^{1}$ \\ ${ }^{1}$ Department of Cardiology, Children's Hospital of Soochow University, Suzhou, China; ${ }^{2}$ Department of Pediatrics, The First People's Hospital \\ of Yan Cheng, Yancheng, China; ${ }^{3}$ Department of Pediatrics, Yi Ji Shan Hospital, Wan Nan Medical College, Wuhu, China; ${ }^{4}$ Institute of Pediatric \\ Research, Children's Hospital of Soochow University, Suzhou, China \\ Contributions: (I) Conception and design: J Qin, H Lv; (II) Administrative support: H Lv, Y Ding; (III) Provision of study materials or patients: H \\ Lv; (IV) Collection and assembly of data: Y Ding, C Huang, M Li, M Hou; (V) Data analysis and interpretation: J Qin, Y Zheng; (VI) Manuscript \\ writing: All authors; (VII) Final approval of manuscript: All authors. \\ \#These authors contributed equally to this work. \\ Correspondence to: Haitao Lv; Guanghui Qian. Institute of Pediatric Research, Children's Hospital of Soochow University, Zhongnan Street, Suzhou \\ 215025, China. Email: lvhaitao@suda.edu.cn; ghqian@suda.edu.cn.
}

Background: Kawasaki disease (KD) is a systemic vasculitis syndrome that commonly occurs in children. Autophagy has been increasingly shown to be involved in various cardiovascular diseases, including endothelial dysfunction and vascular endothelial injury. However, whether autophagy is implicated in the pathogenesis of KD remains poorly understood, and particularly, how the dysfunction of human coronary artery endothelial cells (HCAECs) is associated with autophagy in peripheral blood mononuclear cells (PBMCs) from KD patients awaits further investigation.

Methods: Peripheral blood samples were collected from KD patients, common fever patients, and healthy controls. The PBMC samples were isolated from KD blood samples collected at three different phases: the acute phase before therapy (acute-KD), 1 week (subacute-KD), and 4 weeks (convalescent-KD) after drug administration.

Results: The autophagy flux was significantly increased in the PBMCs of KD patients at acute phase. The PBMCs of acute KD patients could induce autophagy in HCAECs and promote the secretion of chemokines and pro-inflammatory factors after cocultured with HCAECs whereas 3-methyladenine (3-MA) drug could partly reverse this process.

Conclusions: Autophagy is involved in the inflammatory injury of vascular endothelial cells associated with PBMCs in KD patients, and may play a crucial role in regulating inflammation. Hence, we identify a novel regulatory mechanism of vascular injury in this disease.

Keywords: Autophagy; Kawasaki disease (KD); peripheral blood mononuclear cell (PBMC); human coronary artery endothelial cell (HCAEC); inflammatory injury

Submitted Jul 26, 2021. Accepted for publication Nov 25, 2021.

doi: $10.21037 / \mathrm{tp}-21-344$

View this article at: https://dx.doi.org/10.21037/tp-21-344 


\section{Introduction}

Kawasaki disease (KD) is a systemic vasculitis syndrome that affects medium-sized vessels. Although great efforts have been devoted over the past decades, the mechanisms underlying the pathogenesis of $\mathrm{KD}$ remain poorly understood. What is known is that the degree of coronary artery involvement may affect the long-term prognosis of this disease. $\mathrm{KD}$ is a principal cause of acquired coronary disease in pediatric patients, and also a high-risk component to acute coronary syndrome and myocardial infarction in many adults (1).

The activation of immune cells has been reported in $\mathrm{KD}$ patients, and both innate and adaptive immune systems are involved in this process (2-4). Another possible mechanism that may lead to $\mathrm{KD}$ onset is the inflammatory injury of vascular endothelial cells (5). Since endothelial cells constitute an important barrier in blood vessels, they can regulate inflammation and vascular function through a variety of complex mechanisms (6), including the transport of oxygen and energy as well as tumor immune regulation and cellular metabolism $(7,8)$.

Autophagy is an essential biological process in mammals, which plays pivotal roles under both physiological and pathological conditions (9). Autophagy enables the degradation of intracellular components by delivering them into lysosomes, mainly through autophagosome assembly from membrane-bound vesicle intermediates (10). Several studies have demonstrated that autophagy is crucial for sustaining organ growth, immunity and metabolic active balance, and it accomplishes a myriad of functions at the cellular level $(11,12)$. In addition, other studies have reported that autophagic processes are involved in aging, inflammation, nephropathies, metabolic diseases, cardiovascular diseases, neurological disorders, and various cancers $(13,14)$.

In recent years, a better understanding of the relationship between autophagy and inflammation has been rapidly developed $(12,15)$. Autophagy plays a key role in host invasion via several mechanisms. On the one hand, it can regulate antigen presentation, activate acquired immunity, promote lymphocyte differentiation and enhance memory $\mathrm{T}$ cell development. On the other hand, it can modulate cytokine signaling and promote innate immunity $(16,17)$. Growing evidence suggests that autophagy is actively involved in endothelial dysfunction and vascular endothelial injury, which can be reflected in the pathogenesis of atherosclerosis, sepsis and diabetes $(8,18-20)$. Thus, we speculate that autophagy is a crucial factor for peripheral blood mononuclear cells (PBMCs)-related endothelial inflammation. However, there is a lack of studies focusing on the role of autophagy in $\mathrm{KD}$. In this study, we first established a co-culture system of PBMCs from KD patients and human coronary endothelial cells (HCAECs), and then assessed the role of autophagy in KD. We present the following article in accordance with the MDAR reporting checklist (available at https://dx.doi. org/10.21037/tp-21-344).

\section{Methods}

\section{Patients and control subjects}

This study was approved by the Ethics Committee of the Children's Hospital of Soochow University (No. 2021CS176) and was conducted in compliance with the Declaration of Helsinki (as revised in 2013). Written informed consent was obtained from the participants' parents or legal guardians. Patients with a diagnosis of $\mathrm{KD}$ according to the diagnostic criteria established by the American Heart Association guidelines were recruited from Children's Hospital of Soochow University between June 2019 and October 2019. The onset time of all children included in this study was within 7 days, and they were not treated with intravenous immunoglobulin (IVIG) before admission as well as nonsteroidal anti-inflammatory drugs (NSAIDs), steroids or immunosuppressive within 2 weeks after admission. Meanwhile, we excluded children with incomplete KD and other significant medical conditions. None of the participants in this study had coronary artery lesions and serious complications. Patients were treated with $2 \mathrm{~g} / \mathrm{kg}$ IVIG as a single infusion over 10-12 h, together with $30-50 \mathrm{mg} / \mathrm{kg} /$ day acetylsalicylic acid (ASA). After $48 \mathrm{~h}$ of normal body temperature, the doses of ASA were reduced to $3-5 \mathrm{mg} / \mathrm{kg} /$ day. There were 4 cases of resistance to IVIG treatment. Subsequently, we classified all patients into three phases: acute phase (prior to IVIG treatment), subacute phase ( 1 week after IVIG treatment), and convalescent phase (4 weeks after IVIG treatment). Children who had common virus infection with fever symptoms (axillary temperature $>37.5^{\circ} \mathrm{C}$, duration of fever $\geq 3$ days) and those with normal physical examination findings were classified as the fever and control groups, respectively. All groups did not differ significantly in terms of age, gender, and weight. The baseline information of the subjects included in this 
Table 1 The Characteristic features of KD patients and the control participants

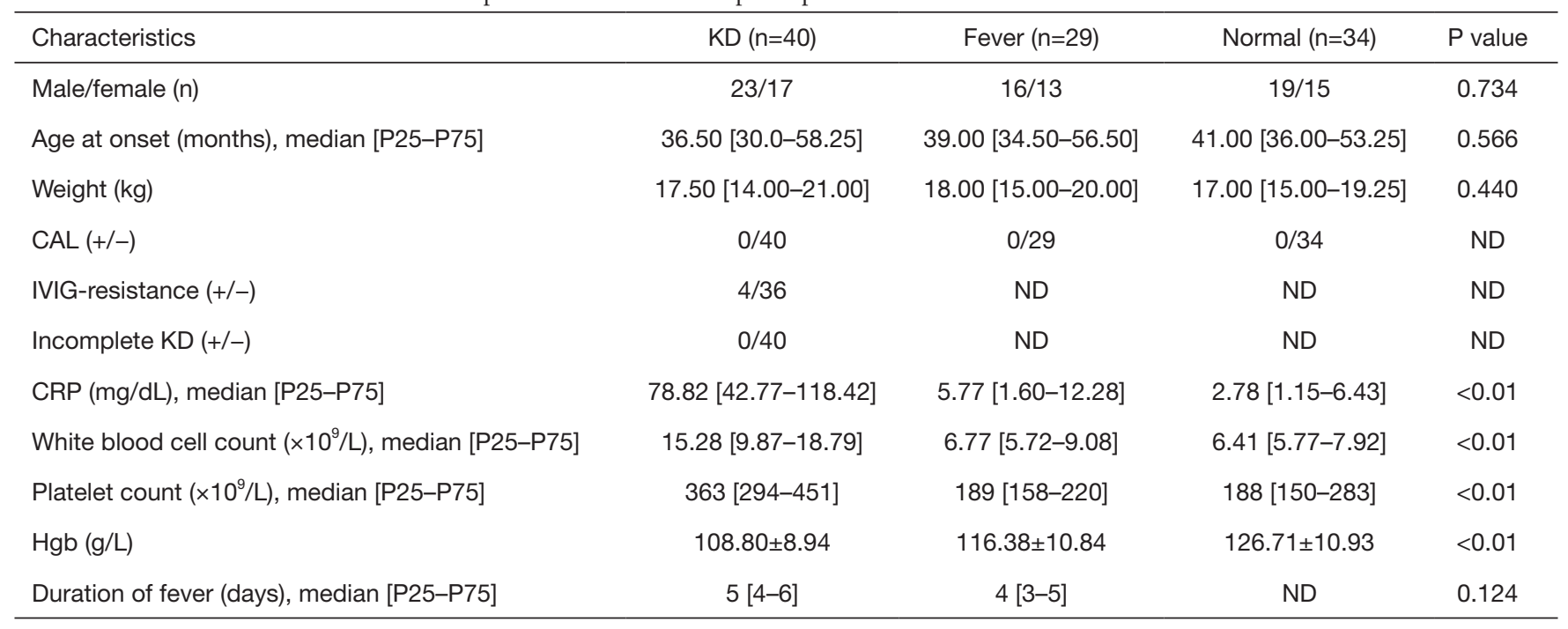

n, number of patients; KD, Kawasaki disease; CAL, coronary arterial lesions; Hgb, the mean hemoglobin concentration normalized for age; CRP, C-reactive protein; ND, no data.

study are shown in Table 1.

\section{Isolation of PBMCs}

Whole blood $(2 \mathrm{~mL})$ was collected from each volunteer who agreed to participate in this experiment. The blood samples were stored in K3 EDTA vacutainer tubes (Greiner VACUETTE, Frickenhausen, Germany). Plasma was obtained from all blood samples within $4 \mathrm{~h}$ after collection. Then, Ficoll lymphocyte separation solution (TBD Science, Inc., Tianjin, China) was used to separate PBMCs through a gradient centrifugation. The isolated PBMCs were cultured or placed in $1.5-\mathrm{mL}$ tubes and stored at $-80{ }^{\circ} \mathrm{C}$ until further use. The PBMCs used for cell culture were counted and then added to RPMI-1640 medium (Gibco, USA) supplemented with $10 \%$ fetal bovine serum (FBS; Gibco, Grand Island, NY, USA) and $200 \mathrm{U} / \mathrm{mL}$ penicillinstreptomycin (Gibco, USA).

\section{Human coronary endothelial cell culture}

HCAECs were purchased from the American Type Culture Collection (ATCC; Manassas, VA, USA). The cells were cultured in endothelial cell medium (ScienCell, San Diego, CA, USA) supplemented with $1 \%$ endothelial cell growth supplement (ECGS), 5\% (v/v) FBS, and 1\% penicillinstreptomycin.

\section{Establishment of co-culture model}

With regard to the co-culture system, Transwell cell culture inserts (Corning, NY, USA) with $0.4-\mu \mathrm{m}$ pore size and 12well plates were used. This type of insert could prevent the migration of cells from the top chamber to the bottom one. Briefly, HCAECs $\left(1 \times 10^{5}\right.$ cells/well $)$ were seeded in 12 -well plates, and then incubated at $37{ }^{\circ} \mathrm{C}$ and $5 \% \mathrm{CO}_{2}$ for $6 \mathrm{~h}$ to reach adhesion. Next, PBMCs $\left(2 \times 10^{6}\right.$ cells/well $)$ in $500 \mu \mathrm{L}$ complete media were seeded into the upper chamber of the Transwell inserts for each of the 12 wells containing HCAECs. The cells were co-cultured in a $5 \%$ $\mathrm{CO}_{2}$ incubator at $37^{\circ} \mathrm{C}$ for $48 \mathrm{~h}$.

\section{Western blot analysis}

After co-culturing for $48 \mathrm{~h}$, the cells were washed three times with cold PBS. To extract proteins, HCAECs were harvested and lysed in RIPA cell lysis buffer (Beyotime, Haimen, China) pre-chilled at $4{ }^{\circ} \mathrm{C}$. Bradford method (Sangon Biotech, Shanghai, China) was employed to measure the concentration of total protein in the lysates. Approximately $20 \mu \mathrm{g}$ lysate protein was loaded into each well in $12.5 \%$ sodium dodecyl sulfate-poly acrylamide gels, and then separated by electrophoresis at $100 \mathrm{~V}$. Next, the proteins were transferred onto polyvinylidene fluoride membranes (PVDF, $0.22 \mu \mathrm{m}$; Bio-Rad, CA, USA). After 
blocking with $5 \%$ nonfat milk solution on a shaker at room temperature for $2 \mathrm{~h}$, the membranes were incubated with a primary antibody against light chain 3 II/I (LC3 II/I) (1:1,000; Cat: 4108; Cell Signaling Technology (CST), Danvers, $\mathrm{MA}, \mathrm{USA}$ ) at $4{ }^{\circ} \mathrm{C}$ with gentle shaking for $14-16 \mathrm{~h}$. After washing with $\mathrm{PBS}$, the membranes were incubated with a secondary antibody (1:5,000; Cat: 7074; CST, USA) at room temperature for $1 \mathrm{~h}$. Protein bands were detected using an electrochemiluminescence (ECL) reagent (EpiZyme, Shanghai, China) for development, and then visualized on an Image Quant LAS 4000 imaging system. The blots were also probed against GAPDH (1:5,000; Cat: ab9485; Abcam, Cambridge, MA, USA), which was used as an internal loading control.

\section{Immunocytochemistry}

HCAECs were seeded in confocal microscopy plates (diameter: $35 \mathrm{~mm}$; glass bottom diameter: $20 \mathrm{~mm}$ ) at a density of $5 \times 10^{3}$ cells per well and cultivated as described above. Meanwhile, PBMCs were grown in the upper chamber of the Transwell insert (PET $0.4 \mu \mathrm{m}$, available for 6-well plate). After $48 \mathrm{~h}$ of co-cultivation with PBMCs, HCAECs were immediately fixed with cold $4 \%$ paraformaldehyde for $30 \mathrm{~min}$, washed with PBS $(5 \mathrm{~min} \times 3)$, permeabilized with $0.5 \%$ Triton $\mathrm{X}-100$, and blocked with $1 \%$ bovine serum albumin (BSA) for $30 \mathrm{~min}$. Subsequently, the cells were incubated overnight with anti-LC3 II/I antibody (1:200; Cat: 4108; CST, USA) at a dilution of 200 times in $0.5 \%$ BSA. After three times of gentle washing with PBS, the cells were incubated with Alexa Fluor 594-conjugated goat anti-rabbit IgG antibody (1:200; Cat: A-11012; Invitrogen, Carlsbad, CA, USA). Nuclei were identified by DAPI staining. Olympus FV1000 (IX81) confocal microscope (Tokyo, Japan) was used to capture the fluorescent images.

\section{Transmission electron microscopy (TEM)}

Whole blood (2 mL) was collected using the anticoagulant blood tubes without EDTA, followed by PBMC isolation. After washing with PBS and centrifugation at 1,000 rpm, PBMCs were deposited at the bottom of the $1.5-\mathrm{mL}$ EP tube to form a mass. Then, $2.5 \%$ glutaraldehyde $(\mathrm{pH} 7.4)$ was added and fixed at $4{ }^{\circ} \mathrm{C}$ for $24 \mathrm{~h}$. The fixed cells were embedded in agarose with low melting point. After washing three times with $0.1 \mathrm{M}$ phosphate buffer ( $\mathrm{pH} 7.2$ ), the cells were fixed in $1 \%$ osmic acid at $4{ }^{\circ} \mathrm{C}$ for $2 \mathrm{~h}$, followed by gradient dehydration with a graded series of ethanol. Subsequently, the samples were embedded in Epon-Araldite resin for penetration, and then placed in a model for polymerization. Later, the ultrathin sections were collected for microstructure analysis. After counterstaining with $3 \%$ uranyl acetate and $2.7 \%$ lead citrate, the sections were examined using a HT7800 TEM.

\section{ELISA analysis}

After 48 h of co-cultivation, HCAECs were lysed by repeated freezing and thawing, PBS rinsing, and ultrasonication. The protein concentration was measured with Bradford method. The supernatant was obtained to determine the levels of tumor necrosis factor $\alpha$ (TNF- $\alpha$ ), interleukin 6 (IL-6), interleukin 8 (IL-8), intercellular adhesion molecule 1 (ICAM-1), and vascular cell adhesion molecule 1 (VCAM-1). For each experimental group, the cells were seeded in five independent wells, and the assay was performed in triplicate. ELISA kits (Dakewe Biotech Co., Ltd., Shenzhen, China) were used in accordance with the manufacturer's instructions. The optical density (OD) was measured at $450 \mathrm{~nm}$ using a microplate reader (Thermo Scientific, USA).

\section{Statistical analysis}

Each experiment was repeated three or more times. Statistical analyses were performed with SPSS 21.0. GraphPad Prism 7 was used to construct the figures. All data were expressed as mean \pm standard error of the mean (SEM). To compare the differences between groups, oneway analysis of variance (ANOVA) and Student's $t$-tests were used as appropriate. $\mathrm{P}<0.05$ was considered statistically significant.

\section{Results}

\section{PBMC autophagy is significantly induced in KD patients}

Eleven blood samples were collected from six KD patients and five healthy controls in the first 15 days. The samples were collected from each patient at three different phases, including acute, subacute, and convalescent phases. To assess autophagy in the PBMCs of KD patients, we evaluated LC3 protein levels and examined autophagy-related changes via TEM analysis. Since the formation of LC3 II from the cytosolic LC3 I is recognized as a key readout for mature 

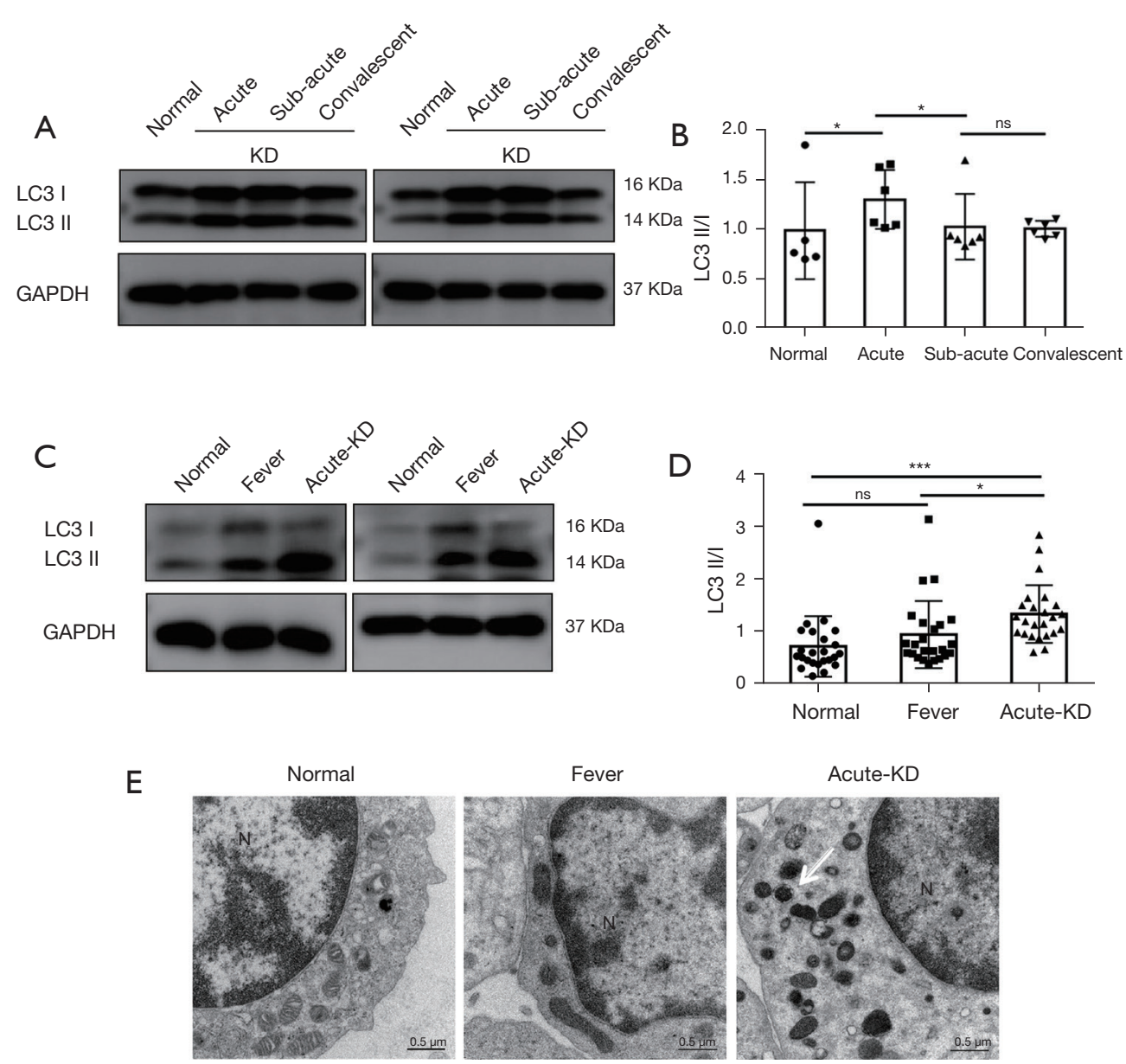

Figure 1 Autophagy is induced in the PBMCs of KD patients. (A) Representative blots of PBMCs in KD patients at acute, subacute and convalescent phases (control: $\mathrm{n}=5, \mathrm{KD}: \mathrm{n}=6$ ). All samples were subjected to immunoblot analysis with specific antibodies against LC3 II/I and GAPDH. (B) Western blots of PBMCs in KD, fever and control groups ( $\mathrm{n}=24$ per group). All samples were subjected to immunoblot analysis with specific antibodies against LC3 II/I and GAPDH. (C,D) The expression levels of LC3 in (A,B) were quantified by densitometry, and the level of LC3 II/I was analyzed. (E) TEM examination of PBMCs in KD, fever and control groups. The arrowhead indicates the autophagosome. *, $\mathrm{P} \leq 0.05$; **, $\mathrm{P} \leq 0.001$; ns, $\mathrm{P}>0.05$. KD, Kawasaki disease; PBMC, peripheral blood mononuclear cell; LC3, light chain 3 .

autophagosome formation, we determined the levels of these proteins by Western blotting. The results revealed that $\mathrm{KD}$ patients at acute phase exhibited higher proportions of LC3 II and LC3 I than those at subacute and convalescent phases (Figure 1A,1B). Subsequently, we isolated PBMCs from 24 patients with acute $\mathrm{KD}$, and compared the levels of LC3 with healthy controls and common fever patients. The results showed that the ratio of LC3 II/I in acute KD patients was significantly increased compared with other groups (Figure 1C,1D). From the TEM images, we observed autophagosomes and mitochondrial vacuoles in the PBMCs of acute $\mathrm{KD}$ patients. However, these alterations were not observed in both control and fever groups (Figure 1E). Collectively, these results indicate that autophagy is induced in the PBMCs of KD patients.

\section{HCAEC autophagy is triggered by the PBMCs of acute KD patients in the co-culture model}

After co-culturing of HCAECs with PBMCs for $48 \mathrm{~h}$, HCAEC morphology did not change significantly among the three groups (Figure 2A). However, the ratio of LC3 II/I in endothelial cells co-incubated with PBMCs from $\mathrm{KD}$ patients was significantly higher compared with the 

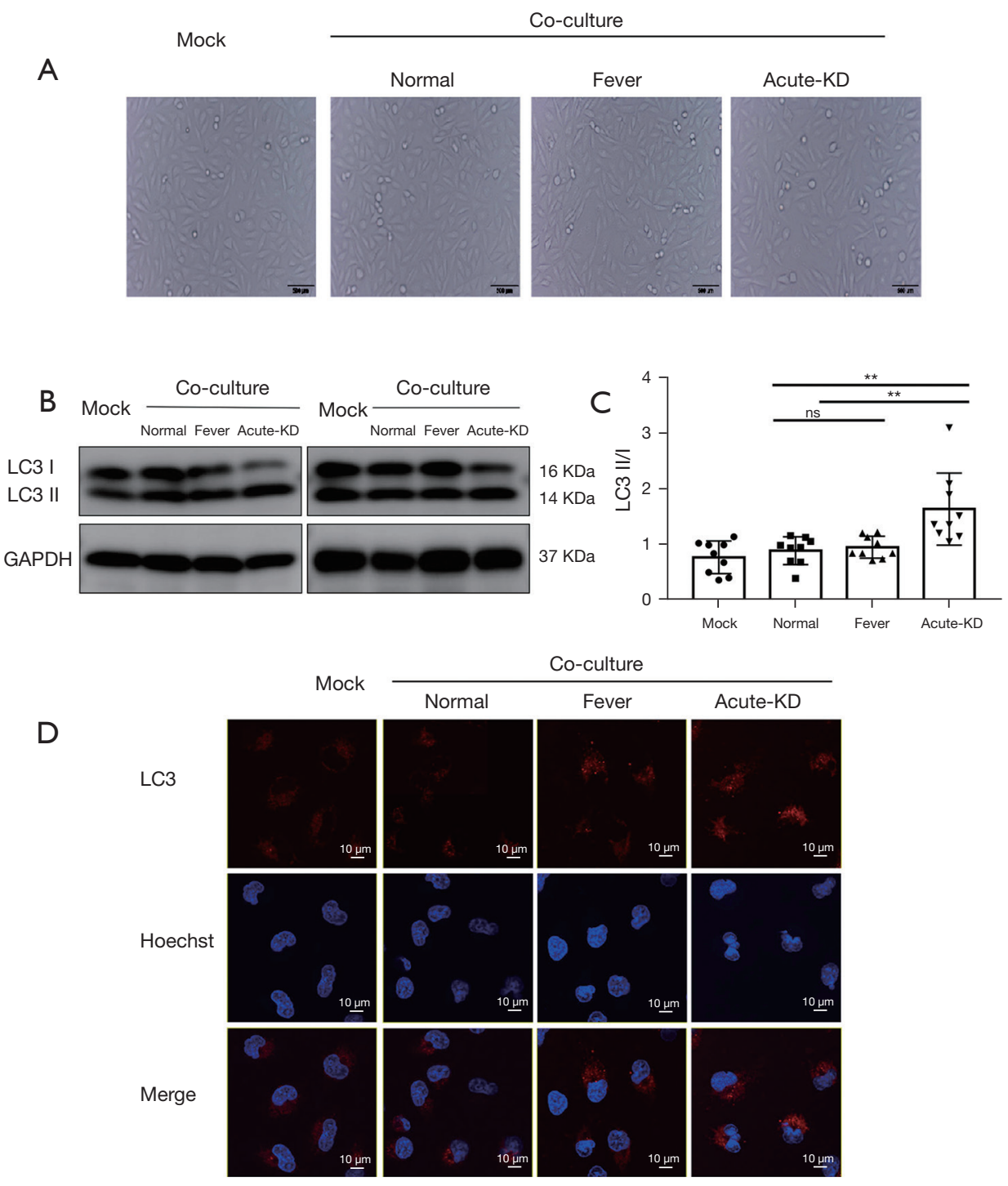

Figure 2 HCAECs autophagy is triggered by PBMCs of acute KD patients in the co-culture model. (A) HCAECs did not change their shape after co-culturing with the PBMCs of KD patients for $48 \mathrm{~h}$ (scale bar, $500 \mu \mathrm{m}$ ). (B) HCAECs incubated with PBMCs from acute KD, fever and control groups ( $\mathrm{n}=3$ per group) for $48 \mathrm{~h}$, and then subjected to immunoblot analysis with specific antibodies against LC3 II/I and GAPDH. (C) The expression levels of LC3 were quantified by densitometry via Image J, and the level of LC3 II/I was analyzed. (D) The distribution of LC3 in HCAECs after co-incubation with PBMCs from acute KD, fever and control groups for $48 \mathrm{~h}$, which was detected by immunofluorescence ( $\times 600$ ). All experiments were repeated three times independently. ${ }^{* *}, \mathrm{P} \leq 0.01$; ns, $\mathrm{P}>0.05$. HCAEC, human coronary endothelial cell; KD, Kawasaki disease; PBMC, peripheral blood mononuclear cell; LC3, light chain 3.

fever and control groups, as revealed by Western blotting (Figure 2B,2C). Moreover, we observed evident changes in LC3 levels using the immunofluorescence method. The expression level of LC3 was significantly increased in HCAECs co-incubated with PBMCs from KD patients compared with the other two groups (Figure 2D). These results indicate that the PBMCs of KD patients can enhance autophagy flux in HCAECs.

\section{Co-cultured with PBMCs of KD patients promote the secretion of chemokines and inflammatory cytokines in HCAECs}

It is well known that endothelial cells secrete cytokines 

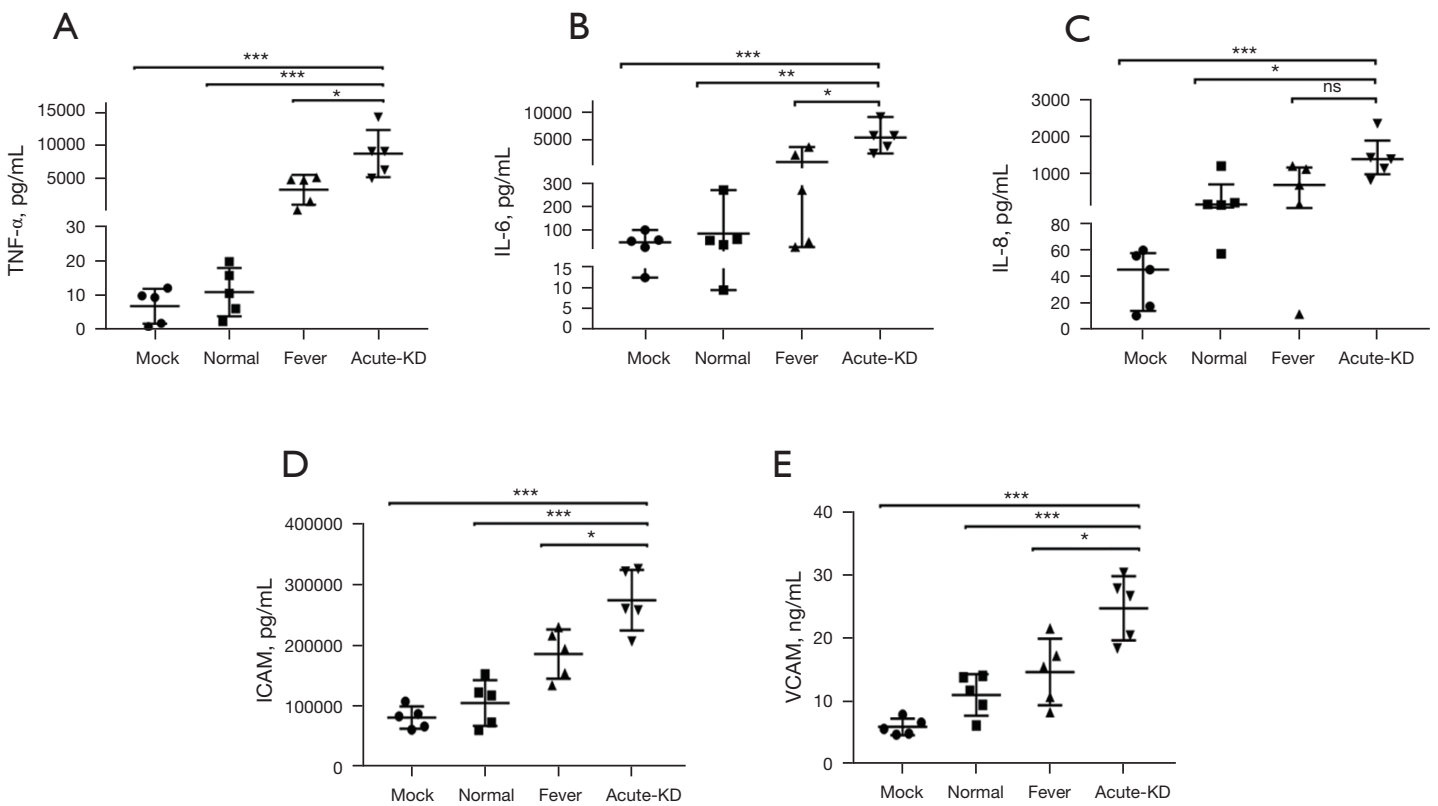

Figure 3 The PBMCs of acute KD patients promote the secretion of inflammatory cytokines and chemokines in HCAECs. (A-C) Expression levels of TNF- $\alpha$, IL-6 and IL-8 in HCAECs after co-culture. (D,E). Expression levels of ICAM and VCAM in HCAECs after co-culture. All experiments were repeated three times independently. *, $\mathrm{P} \leq 0.05$; ${ }^{* *}, \mathrm{P} \leq 0.01$; **, $\mathrm{P} \leq 0.001$; ns, $\mathrm{P}>0.05$. KD, Kawasaki disease; PBMC, peripheral blood mononuclear cell; HCAEC, human coronary endothelial cell; TNF- $\alpha$, tumor necrosis factor $\alpha$; IL-6, interleukin 6; IL-8, interleukin 8; ICAM, intercellular cell adhesion molecule; VCAM, vascular cell adhesion molecule.

(e.g., chemokines, TNF- $\alpha$, IL-6 and IL-8), and these inflammatory-related factors are significantly increased in the plasma of KD patients. Therefore, we lysed HCAECs that were co-cultured with PBMCs for $48 \mathrm{~h}$ via ultrasonic disruption in order to determine the concentration of these inflammatory-related factors in our co-culture system. The levels of TNF- $\alpha$ and IL-6 in HCAECs co-incubated with PBMCs from KD patients were significantly higher than those in the control and fever groups (Figure 3A,3B). In addition, the level of IL- 8 in endothelial cells cocultured with PBMCs from KD patients was significantly higher than that in the control group, while there was no significant difference when compared with that in the fever group (Figure 3C). Activation of endothelial cells plays an important role in the development of KD. Therefore, we examined the expression levels of ICAM and VCAM in co-cultured endothelial cells. As expected, HCAECs cocultured with PBMCs from KD patients show an increased expression level of ICAM-1 and VCAM-1 compared with the other two groups (Figure 3D,3E).

\section{3-methyladenine (3-MA) inbibits the secretion of chemokines and inflammatory cytokines in HCAECs co-cultured with PBMCs from KD patients}

Finally, to verify the role of autophagy in our co-culture system, we co-cultured HCAECs with PBMCs from five patients with acute KD and investigated the effects of 3-MA on cytokine secretion. The PBMCs of each patient were divided into two groups, and two identical co-culture systems were constructed, in one of which the HCAECs were pretreated with 3-MA $(5 \mathrm{mM})$ for $1 \mathrm{~h}$. After $48 \mathrm{~h}$ of co-cultivation, the adherent HCAECs were collected from the lower wells. The levels of TNF- $\alpha$, IL-6, and IL-8 in 3-MA-pretreated HCAECs were significantly lower than those in the untreated group, indicating that 3-MA could inhibit the secretion of inflammatory cytokines in HCAECs co-cultured with PBMCs from acute KD patients (Figure $4 A-4 C$ ). With regard to endothelial cell activation, the increased expression levels of ICAM- 1 and VCAM- 2 in HCAECs were also suppressed by 3-MA treatment when 

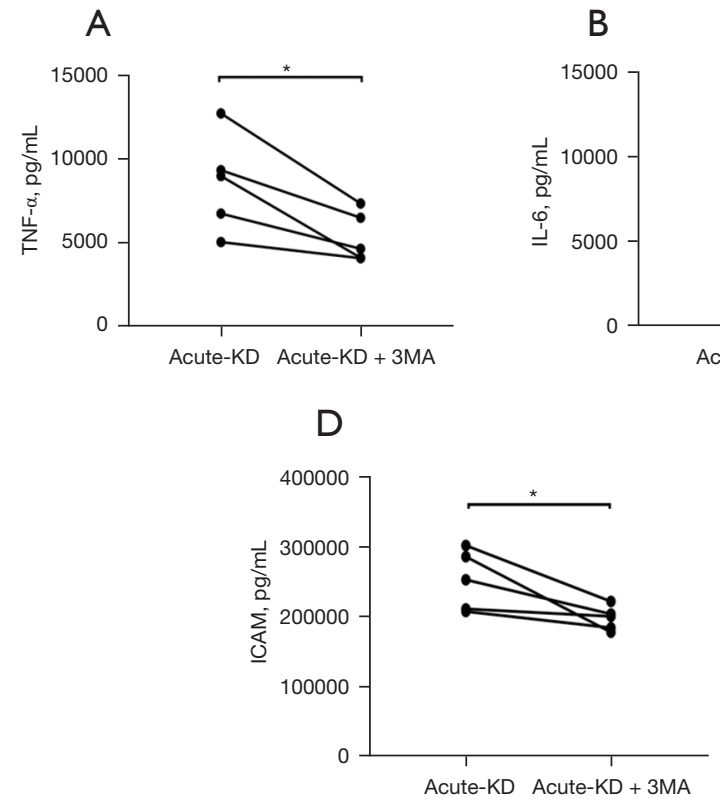
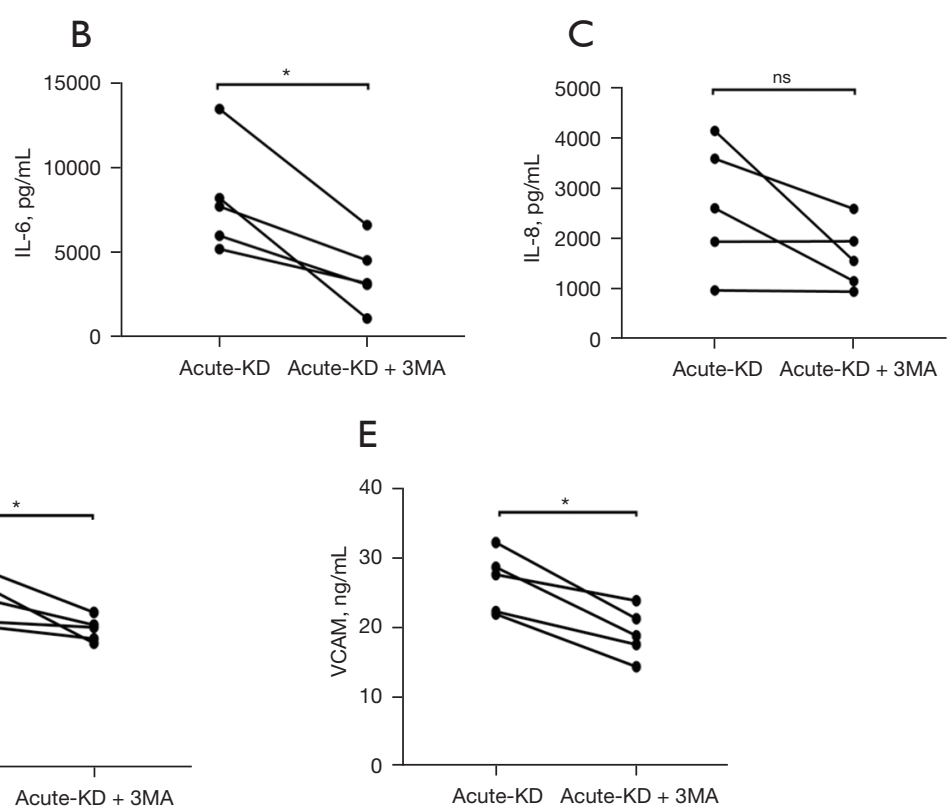

Figure 4 3-MA inhibits the expression of inflammatory cytokines in HCAECs during co-cultivation. (A-C) 3-MA decreases the expression levels of TNF- $\alpha$, IL-6 and IL-8 in HCAECs during co-cultivation. (D,E) 3-MA reduces the expression levels of ICAM and VCAM in HCAECs during co-cultivation. All experiments were repeated three times independently. * $\mathrm{P} \leq 0.05$; ns, $\mathrm{P}>0.05$. 3-MA, 3-methyladenine; HCAEC, human coronary endothelial cell; TNF- $\alpha$, tumor necrosis factor $\alpha$; IL-6, interleukin 6; IL-8, interleukin 8; ICAM, intercellular cell adhesion molecule; VCAM, vascular cell adhesion molecule.

compared to the untreated cells (Figure $4 D, 4 E$ ).

\section{Discussion}

In a normal heart, autophagy is a dynamic process at the subcellular level. From a physiological viewpoint, autophagy can remove aging-related compounds, damaged substrates or excess substances in cells through lysosomes. There are three principal forms of autophagy: microautophagy, chaperone-mediated autophagy, and macroautophagy. Both redox-active protein aggregates and cytoplasmic bacteria can be eliminated via autophagy, indicating that exogenous as well as endogenous substances can be degraded (21). Autophagosomes form a bilayer membrane structure that engulfs isolated cellular contents, which is then combined with lysosomes to complete the process of autophagy (22). LC3 family proteins are key components for autophagosome formation. An increased proportion of LC3 II or an accumulation of autophagosome is indicative of the induced autophagy flux (23). This basic autophagy maintains the physiological balance of the heart. However, the role of autophagy in cardiovascular disease and inflammation is quite complex. A previous study has shown that although autophagy is capable of preventing spontaneous/ endogenous inflammation, its persistent dysregulation can induce pathological changes and ultimately lead to tissue damage (24). Therefore, we evaluated autophagy by gauging the ratio of LC3 II/I.

$\mathrm{KD}$-induced cardiac and vascular inflammation can activate macrophages, neutrophils and monocytes, which further invade vascular endothelium and last for several days $(25,26)$. Thus, it is of great clinical value to explore the molecular mechanisms underlying the inflammatory injury of vascular endothelial cells from the perspective of peripheral blood cells. The co-culture system can serve as a novel model to investigate the effect of PBMCs on endothelial cells. In this study, we found that PBMC autophagy was increased in KD patients at the acute phase, which was different from the autophagy of leukocytes dominated by neutrophils as reported previously (27). This implies that PBMCs and neutrophils play differential roles in KD. Previous studies have shown that infection is indeed involved in the pathogenesis of $\operatorname{KD}(28,29)$. A novel RNA virus, which was detected in the bronchial epithelial cells and other cells of patients, could be linked to the onset of KD (3,30-32). Rowley proposed that an unknown 
virus could infect the respiratory tract of individuals with a certain genetic background, which then entered the bloodstream through macrophages and invaded coronary vessels (29). These findings reveal that peripheral blood cells have a new way to participate in vascular endothelial cell injury.

Vascular endothelial cells play a central role in sustaining the physiological functions of the cardiovascular system. Clinical studies have confirmed that endothelial dysfunction is a common risk factor in various cardiovascular diseases (33). However, the role of autophagy in human vascular endothelial cells has not been fully elucidated. A channel was provided by the co-culture model of PBMCs from KD patients and endothelial cells to examine the roles of autophagy in KD. The smaller pore size of the Transwell inserts membrane prevented the migration of PBMCs in the upper layer and inhibited a direct cell-cell contact between top and bottom layers. However, cytokine-mediated cell communication is existed, and this may be the inducing factor for endothelial cell inflammation. Different from the autophagy inhibition of endothelial cells after TNF- $\alpha$ stimulation for $2 \mathrm{~h}$ (34), we observed that PBMCs from KD patients could induce endothelial cell autophagy in a coculture system for $48 \mathrm{~h}$, and this inconsistency also reflected the complexity of autophagy. Simultaneously, PBMCs from $\mathrm{KD}$ patients induced an increase in the levels of ICAM, VCAM, and pro-inflammatory factors (e.g., TNF- $\alpha$, IL-6 and IL-8) in endothelial cells. This provides direct evidence for the inflammatory injury of endothelial cells induced by PBMCs from KD patients.

Autophagy of endothelial cells that are induced by different biological and chemical factors may lead to different outcomes (20). Certain naturally occurring compounds, such as analogs of resveratrol (34), epigallocatechin gallate (35), and curcumin (36), have shown to exhibit cardiovascular protective effects by inducing autophagy. On the contrary, cigarette smokeinduced autophagy appears to be damaging and promote cell death (37). In the co-culture system, the outcome of endothelial cells was changed by 3-MA, an autophagy inhibitor, and the inflammatory injury of endothelial cells induced by PBMCs was suppressed. This regulation on inflammatory factor secretion differs from the basic inhibitory effect on autophagy, suggesting that autophagy may play a pro-inflammatory role when the inflammasome is activated $(38,39)$. Furthermore, we speculate that the cytokines secreted by the PBMCs of KD patients can induce excessive autophagy in endothelial cells, and are involved in the inflammatory damage of HCAEC in the inflammatory damage of blood vessels. This also corroborates that the systemic inflammatory response may potentiate the inflammatory response in coronary lesions, leading to endothelial dysfunction (40). Additionally, the PBMCs in the co-culture system may also be affected by 3-MA. However, many studies have shown that the loss of autophagy in $\mathrm{T}$ cells and B cells can promote an inflammatory response in the body $(41,42)$, which adds complexity and confusion to the results of our study. In the follow-up experiments, we will attempt to use the culture supernatant of PBMCs from KD patients as a stimulant to induce the inflammatory injury of vascular endothelial cells in order to get more direct evidence.

There are several limitations to this study. First, the amount of KD cases was small, and the evaluated time points of cases were scarce. Second, we did not elucidate the mechanisms underlying the relationship between autophagy and vascular inflammation in KD patients, which needs to be investigated in the follow-up study. Third, we neglected the effects of autophagy on diverse phenomena and its roles in different categories of $\mathrm{KD}$ such as complete and incomplete KD, IVIG responding and non-responding, or in the presence and absence of coronary artery abnormalities. Further studies with larger sample sizes and long-term follow-up periods are warranted to provide more definitive evidence.

\section{Conclusions}

In summary, we found the autophagy flux was induced in PBMCs of KD patients, and identified that the PBMCs of acute KD patients could cause the inflammatory injury of vascular endothelial cells by using the co-culture method. Furthermore, such inflammatory injury could be ameliorated by adding the 3-MA. These findings suggest that autophagy occurs in the PBMCs of KD patients and is associated with the inflammatory injury of vascular endothelial cells, which provide a novel mechanism of vascular injury in this disease and its potential therapeutic target.

\section{Acknowledgments}

The authors give thanks to Ying Liu for consultation at the start of the study on the design and implementation of the trial. In addition, we would like to show our gratitude to all of the participants for their involvement in this study. The 
authors would like to express their gratitude to EditSprings for the expert linguistic services provided.

Funding: This work was supported by the grants from the National Natural Science Foundation of China (No. 81971477, No. 81870365, No. 82070512, No. 81970436), Jiangsu Provincial Medical Young Talents (QNRC2016756), the Applied Foundational Research of Medical and Health Care of Suzhou City (SYS2019086), Suzhou Medical Key Discipline Pediatric Cardiology (Szxk201507), Key Medical Talents in Jiangsu Province (ZDRCA2016049), The second cycle key subjects of maternal and child health in Jiangsu Province (FXK201740), Introduction Project of Clinical Medical Expert Team in Suzhou City (SZYJTD201805), Gusu Health Talent Program (GSWS2020038), Maternal and Child Health Project of Jiangsu Province (No. F201650).

\section{Footnote}

Reporting Checklist: The authors have completed the MDAR reporting checklist. Available at https://dx.doi. org/10.21037/tp-21-344

Data Sharing Statement: Available at https://dx.doi. org/10.21037/tp-21-344

Conflicts of Interest: All authors have completed the ICMJE uniform disclosure form (available at https://dx.doi. org/10.21037/tp-21-344). The authors have no conflicts of interest to declare.

Ethical Statement: The authors are accountable for all aspects of the work in ensuring that questions related to the accuracy or integrity of any part of the work are appropriately investigated and resolved. This study was approved by the Ethics Committee of the Children's Hospital of Soochow University (No. 2021CS176) and was conducted in compliance with the Declaration of Helsinki (as revised in 2013). Written informed consent was obtained from the participants' parents or legal guardians.

Open Access Statement: This is an Open Access article distributed in accordance with the Creative Commons Attribution-NonCommercial-NoDerivs 4.0 International License (CC BY-NC-ND 4.0), which permits the noncommercial replication and distribution of the article with the strict proviso that no changes or edits are made and the original work is properly cited (including links to both the formal publication through the relevant DOI and the license). See: https://creativecommons.org/licenses/by-nc-nd/4.0/.

\section{References}

1. Burns JC, Shike H, Gordon JB, et al. Sequelae of Kawasaki disease in adolescents and young adults. J Am Coll Cardiol 1996;28:253-7.

2. Lin CY, Lin CC, Hwang B, et al. Serial changes of serum interleukin-6, interleukin-8, and tumor necrosis factor alpha among patients with Kawasaki disease. J Pediatr 1992;121:924-6.

3. Rowley AH, Baker SC, Shulman ST, et al. Ultrastructural, immunofluorescence, and RNA evidence support the hypothesis of a "new" virus associated with Kawasaki disease. J Infect Dis 2011;203:1021-30.

4. McCrindle BW, Rowley AH, Newburger JW, et al. Diagnosis, Treatment, and Long-Term Management of Kawasaki Disease: A Scientific Statement for Health Professionals From the American Heart Association. Circulation 2017;135:e927-99.

5. Jia C, Zhang J, Chen H, et al. Endothelial cell pyroptosis plays an important role in Kawasaki disease via HMGB1/ RAGE/cathespin B signaling pathway and NLRP3 inflammasome activation. Cell Death Dis 2019;10:778.

6. Wu KK. Endothelial cells in hemostasis, thrombosis, and inflammation. Hosp Pract (Off Ed) 1992;27:145-50, 152, 163-6.

7. Uldry E, Faes S, Demartines N, et al. Fine-Tuning Tumor Endothelial Cells to Selectively Kill Cancer. Int J Mol Sci 2017;18:1401.

8. Zecchin A, Borgers G, Carmeliet P. Endothelial cells and cancer cells: metabolic partners in crime? Curr Opin Hematol 2015;22:234-42.

9. Tang C, Livingston MJ, Liu Z, et al. Autophagy in kidney homeostasis and disease. Nat Rev Nephrol 2020;16:489-508.

10. Parzych KR, Klionsky DJ. An overview of autophagy: morphology, mechanism, and regulation. Antioxid Redox Signal 2014;20:460-73.

11. Choi AM, Ryter SW, Levine B. Autophagy in human health and disease. N Engl J Med 2013;368:651-62.

12. Levine B, Mizushima N, Virgin HW. Autophagy in immunity and inflammation. Nature 2011;469:323-35.

13. Kimura T, Isaka Y, Yoshimori T. Autophagy and kidney inflammation. Autophagy 2017;13:997-1003.

14. Levine B, Kroemer G. Biological Functions of Autophagy Genes: A Disease Perspective. Cell 2019;176:11-42.

15. Deretic V, Klionsky DJ. Autophagy and inflammation: A 
special review issue. Autophagy 2018;14:179-80.

16. Deretic V, Levine B. Autophagy, immunity, and microbial adaptations. Cell Host Microbe 2009;5:527-49.

17. Xu X, Araki K, Li S, et al. Autophagy is essential for effector CD8(+) T cell survival and memory formation. Nat Immunol 2014;15:1152-61.

18. Wang J, Wang WN, Xu SB, et al. MicroRNA-214-3p: A link between autophagy and endothelial cell dysfunction in atherosclerosis. Acta Physiol (Oxf) 2018.

19. Lenoir O, Jasiek M, Hénique C, et al. Endothelial cell and podocyte autophagy synergistically protect from diabetesinduced glomerulosclerosis. Autophagy 2015;11:1130-45.

20. Jiang F. Autophagy in vascular endothelial cells. Clin Exp Pharmacol Physiol 2016;43:1021-8.

21. Antonioli M, Di Rienzo M, Piacentini M, et al. Emerging Mechanisms in Initiating and Terminating Autophagy. Trends Biochem Sci 2017;42:28-41.

22. Axe EL, Walker SA, Manifava M, et al. Autophagosome formation from membrane compartments enriched in phosphatidylinositol 3-phosphate and dynamically connected to the endoplasmic reticulum. J Cell Biol 2008;182:685-701.

23. Liu N, Shi Y, Zhuang S. Autophagy in Chronic Kidney Diseases. Kidney Dis (Basel) 2016;2:37-45.

24. Cloonan SM, Lam HC, Ryter SW, et al. "Ciliophagy": The consumption of cilia components by autophagy. Autophagy 2014;10:532-4.

25. Tsujimoto H, Takeshita S, Nakatani K, et al. Delayed apoptosis of circulating neutrophils in Kawasaki disease. Clin Exp Immunol 2001;126:355-64.

26. Andreozzi L, Bracci B, D'Errico F, et al. A master role for neutrophils in Kawasaki syndrome. Immunol Lett 2017;184:112-4.

27. Huang FC, Huang YH, Kuo HC, et al. Identifying Downregulation of Autophagy Markers in Kawasaki Disease. Children (Basel) 2020;7:166.

28. Takahashi K, Oharaseki T, Yokouchi Y, et al. Kawasaki disease: basic and pathological findings. Clin Exp Nephrol 2013;17:690-3.

29. Rowley AH, Shulman ST. The Epidemiology and Pathogenesis of Kawasaki Disease. Front Pediatr 2018;6:374.

30. Rowley AH, Baker SC, Shulman ST, et al. RNAcontaining cytoplasmic inclusion bodies in ciliated bronchial epithelium months to years after acute Kawasaki disease. PLoS One 2008;3:e1582.
31. Yang B, Xue Q, Guo J, et al. Autophagy induction by the pathogen receptor NECTIN4 and sustained autophagy contribute to peste des petits ruminants virus infectivity. Autophagy 2020;16:842-61.

32. Richetta C, Grégoire IP, Verlhac P, et al. Sustained autophagy contributes to measles virus infectivity. PLoS Pathog 2013;9:e1003599.

33. Lavandero S, Chiong M, Rothermel BA, et al. Autophagy in cardiovascular biology. J Clin Invest 2015;125:55-64.

34. Huang FC, Kuo HC, Huang YH, et al. Anti-inflammatory effect of resveratrol in human coronary arterial endothelial cells via induction of autophagy: implication for the treatment of Kawasaki disease. BMC Pharmacol Toxicol 2017;18:3.

35. Kim HS, Montana V, Jang HJ, et al. Epigallocatechin gallate (EGCG) stimulates autophagy in vascular endothelial cells: a potential role for reducing lipid accumulation. J Biol Chem 2013;288:22693-705.

36. Han J, Pan XY, Xu Y, et al. Curcumin induces autophagy to protect vascular endothelial cell survival from oxidative stress damage. Autophagy 2012;8:812-25.

37. Meng N, Wu L, Gao J, et al. Lipopolysaccharide induces autophagy through BIRC2 in human umbilical vein endothelial cells. J Cell Physiol 2010;225:174-9.

38. Dupont N, Jiang S, Pilli M, et al. Autophagy-based unconventional secretory pathway for extracellular delivery of IL-1 $\beta$. EMBO J 2011;30:4701-11.

39. Kimura T, Jia J, Kumar S, et al. Dedicated SNAREs and specialized TRIM cargo receptors mediate secretory autophagy. EMBO J 2017;36:42-60.

40. Varga Z, Flammer AJ, Steiger P, et al. Endothelial cell infection and endotheliitis in COVID-19. Lancet 2020;395:1417-8.

41. Matsuzawa-Ishimoto Y, Hwang S, Cadwell K. Autophagy and Inflammation. Annu Rev Immunol 2018;36:73-101.

42. Shibutani ST, Saitoh T, Nowag H, et al. Autophagy and autophagy-related proteins in the immune system. Nat Immunol 2015;16:1014-24.

Cite this article as: Qin J, Zheng Y, Ding Y, Huang C, Hou M, Li M, Qian G, Lv H. Co-culture of peripheral blood mononuclear cell (PBMC) and human coronary artery endothelial cell (HCAEC) reveals the important role of autophagy implicated in Kawasaki disease. Transl Pediatr 2021;10(12):3140-3150. doi: 10.21037/tp-21-344 